\title{
Drug Accountability Character Result in Standard Format
}

National Cancer Institute

\section{Source}

National Cancer Institute. Drug Accountability Character Result in Standard Format. NCI

Thesaurus. Code C87868.

The standard character or string for representation and reporting of drug accountability data. 